\title{
Time-resolved photoelectron spectroscopy of proton transfer in the ground state of chloromalonaldehyde: Wave-packet dynamics on effective potential surfaces of reduced dimensionality
}

\author{
Márcio T. do N. Varella ${ }^{a)}$ \\ Instituto de Física Gleb Wataghin, Universidade Estadual de Campinas, Unicamp, 13083-970 Campinas, \\ São Paulo, Brazil \\ Yasuki Arasaki and Hiroshi Ushiyama \\ Department of Basic Science, Graduate School of Arts and Sciences, University of Tokyo, Komaba, 153- \\ 8902 Tokyo, Japan \\ Vincent McKoy \\ Laboratory for Molecular Sciences, California Institute of Technology, Pasadena, California 91125 \\ Kazuo Takatsuka \\ Department of Basic Science, Graduate School of Arts and Sciences, University of Tokyo, Komaba, 153- \\ 8902 Tokyo, Japan
}

(Received 14 July 2005; accepted 8 March 2006; published online 17 April 2006)

\begin{abstract}
We report on a simple but widely useful method for obtaining time-independent potential surfaces of reduced dimensionality wherein the coupling between reaction and substrate modes is embedded by averaging over an ensemble of classical trajectories. While these classically averaged potentials with their reduced dimensionality should be useful whenever a separation between reaction and substrate modes is meaningful, their use brings about significant simplification in studies of time-resolved photoelectron spectra in polyatomic systems where full-dimensional studies of skeletal and photoelectron dynamics can be prohibitive. Here we report on the use of these effective potentials in the studies of dump-probe photoelectron spectra of intramolecular proton transfer in chloromalonaldehyde. In these applications the effective potentials should provide a more realistic description of proton-substrate couplings than the sudden or adiabatic approximations commonly employed in studies of proton transfer. The resulting time-dependent photoelectron signals, obtained here assuming a constant value of the photoelectron matrix element for ionization of the wave packet, are seen to track the proton transfer. (C) 2006 American Institute of Physics.
\end{abstract}

[DOI: $10.1063 / 1.2191852$ ]

\section{INTRODUCTION}

Time-resolved photoelectron spectroscopy offers several technical and conceptual advantages as a probe of femtosecond wave-packet dynamics and its use is becoming widespread in such studies. ${ }^{1-7}$ In contrast to other probes there are no dark states and the method is well suited for following the dynamics along all energetically allowed internuclear distances simultaneously in a pump-probe experiment. When these photoelectron spectra are angle resolved, they can provide additional insight into the underlying wave-packet dynamics. ${ }^{8-16}$

We have previously developed a formulation for calculating the energy-and angle-resolved photoelectron spectra for femtosecond pump-probe ionization of wave packets in molecules ${ }^{11}$ and reported on its application to wave packets on the double minimum potential in $\mathrm{Na}_{2}$ (Refs. 11 and 17) and on the nonadiabatically coupled ionic $\left(\mathrm{Na}^{+} \mathrm{I}^{-}\right)$and covalent (NaI) states of sodium iodide. ${ }^{14}$ Seideman and coworkers have also developed a theoretical framework for calculating these time-resolved photoelectron spectra, originally

${ }^{a)}$ Electronic mail: mvarella@ifi.unicamp.br for linear systems ${ }^{12}$ and subsequently for systems of arbitrary symmetry, ${ }^{13}$ and have exploited these frameworks to study molecular alignment, ${ }^{18}$ rotation-vibration coupling in $\mathrm{NO},{ }^{19}$ and internal conversion dynamics in pyrazine using the two-dimensional (2D) (two modes) model of Kühl and Domcke. ${ }^{20}$ These diverse applications, which employed $a b$ initio photoionization matrix elements, illustrate the significant dynamical content of time-resolved photoelectron spectra.

In this paper we seek to extend our formulation of timeresolved photoelectron spectroscopy to studies of the dynamics of intramolecular proton transfer. Proton transfer is one of the most important and ubiquitous reactions in both chemistry and biology and has been widely studied. ${ }^{21-26}$ Most theoretical studies have focused on tunneling splitting in the ground state ${ }^{27-37}$ but proton transfer in electronically excited states has also been studied. ${ }^{38-44}$ Spectroscopic studies of the real-time dynamics of proton transfer have been limited, to our knowledge, to excited electronic states. ${ }^{45-47}$ In an application to $o$-hydroxybenzaldehyde and related compounds, Lochbrunner et al. demonstrated the utility of pump-probe photoelectron spectroscopy for probing the dynamics of intramolecular proton transfer in excited states. ${ }^{5}$ We recently 
proposed a femtosecond dump-probe photoelectron spectroscopic scheme for real-time observation of proton transfer in chloromalonaldehyde based on a model study. ${ }^{48}$ The asymmetry of the potential of chloromalonaldehyde conveniently enables distinction of wave packets on different sides of the transition state. In that study, which assumed constant values for the photoionization matrix element over each well, we explored the dynamics of two-dimensional wave packets in the optically coupled ground, excited, and ionic states and observed that the photoelectron signals could indeed track the proton-transfer dynamics. ${ }^{48}$ However, vibrational energy redistribution and relaxation due to coupling between the proton wave packet and skeletal degrees of freedom were neglected in this model study. In applications the molecular skeleton is generally assumed to be either frozen in a given configuration (sudden approximation) or in its optimal geometry (adiabatic approximation). In the former, the proton motion is viewed as being much faster than skeletal relaxation, while in the latter it is viewed as very slow. Proton motion and skeletal relaxation are actually expected, however, to occur on similar time scales and neither of these approximations would seem appropriate.

The effort associated with applications of our formulation of time-resolved photoelectron spectra ${ }^{11}$ to multidimensional systems scales rapidly with the size of the system and full-dimensional studies of skeletal and photoelectron dynamics can be prohibitive. What is needed for such applications is a framework that can effectively reduce the dimensionality of the dynamical system and into which a description of the photoionization dynamics can be embedded. A well-established approach for reducing the dimensionality of dynamical systems is the Cartesian research surface (CRS) model of Ruf and Miller ${ }^{49}$ and its implementation within the time-dependent Hartree ${ }^{50}$ (TDH) or multiconfiguration TDH (MCTDH) schemes ${ }^{51}$ of Kühn and coworkers. In this approach, a few Cartesian reaction coordinates, usually those undergoing larger displacements, are treated in some sophisticated fashion, while the other vibrational degrees of freedom are viewed as a bath (or substrate) of harmonic oscillators coupled to the chosen reaction coordinates. For applications to proton transfer, a natural choice for the reaction coordinates would be the $(x, y)$ Cartesian coordinates of the proton while the skeletal vibrational modes, as well as the out-of-plane motion of the proton $(z)$, would be viewed as belonging to the substrate. Though the CRS/TDH (CRS/MCTDH) approach has been successfully applied to several strongly bonded systems, ${ }^{50-53}$ its application to time-resolved photoelectron spectra of intramolecular proton transfer for energies around the transition state barrier, where large amplitude motion may arise, could be expected to encounter a number of difficulties due to its underlying harmonic assumption for the bath modes and the timedependent potential of the bath modes on the proton.

In this paper we propose a simple but broadly applicable method for generating effective time-independent potential energy surfaces of reduced dimensionality wherein the coupling between reaction and substrate modes is embedded through averaging over an ensemble of classical trajectories. The effective geometry obtained by this temporal and spatial

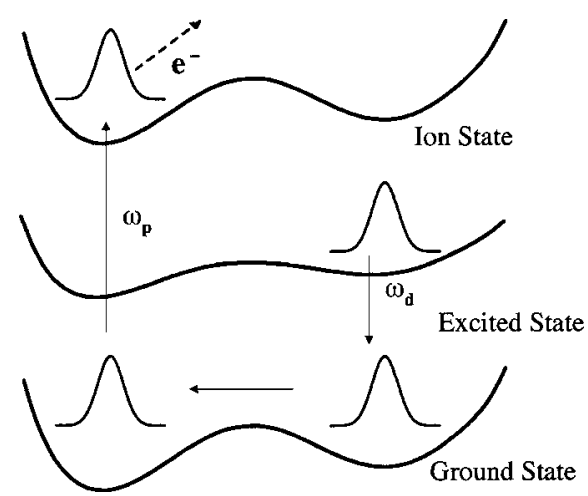

FIG. 1. The dump-probe scheme for chloromalonaldehyde. The initial wave packet in the electronic excited state is dumped to the ground state, where proton transfer is probed.

averaging accounts for proton-substrate coupling in an average sense and should provide a more realistic description of this coupling than the usual sudden or adiabatic approximations for the molecular skeleton. The use of such average geometries leads to a significant reduction in the computational effort in studies of time-resolved photoelectron spectra of multidimensional reactions.

The outline of this paper is as follows. In the next section we describe our formulation of time-resolved photoelectron spectroscopy, focusing on the scaling of the numerical effort in applications to multidimensional systems. We then briefly discuss the difficulties arising in the use of the CRS/ TDH approach in studies of time-resolved photoelectron spectra of the proton-transfer process of interest here. In Sec. III we discuss our scheme for generating potential energy surfaces of reduced dimensionality in which the geometry of the skeleton is averaged over an ensemble of classical trajectories and present results of its application to the electronic ground state of chloromalonaldehyde. We then compare the results of this application to those of the sudden and adiabatic approximations and to the CRS/TDH scheme. In Sec. IV we employ these classically averaged potential surfaces in model studies of the dump-probe photoelectron spectra for intramolecular proton transfer in the electronic ground state of chloromalonaldehyde.

\section{TOWARDS A MULTIDIMENSIONAL TIME- RESOLVED PHOTOELECTRON SPECTROSCOPY FRAMEWORK}

\section{A. Outline of time-resolved dump-probe photoelectron spectroscopy}

The formulation of time-resolved photoelectron spectroscopy employed in these studies has been discussed elsewhere $^{11}$ and only a few working expressions will be given here. Furthermore, because this formulation was originally developed for molecules of arbitrary symmetry, we here focus on the scaling of the computational effort in applications to multidimensional systems. We consider the dump-probe scheme of Fig. 1, where a linearly polarized pulse of frequency $\omega_{d}$ dumps a wave packet from an excited electronic state to the ground state, where it is subsequently ionized by a second time-delayed $(\Delta T)$ linearly polarized 
pulse of frequency $\omega_{p}$. The time evolution of the system is governed by the following set of coupled equations: ${ }^{11,54}$

$$
\begin{aligned}
i \hbar \frac{\partial}{\partial t} \chi_{g}(\mathbf{R}, t)= & {\left[T_{N}+V_{g}(\mathbf{R})\right] \chi_{g}(\mathbf{R}, t) } \\
& +V_{d}(t) \mu_{g e}(\mathbf{R}) \chi_{e}(\mathbf{R}, t) \\
& +V_{p}(t-\Delta T) \sum_{j=1}^{N_{k}} \sum_{l m} C_{l m}^{*}\left(\mathbf{R}, k_{j}, \Omega_{R}, \theta_{P}\right) \\
& \times \chi_{k_{j} l m}(\mathbf{R}, t), \\
i \hbar \frac{\partial}{\partial t} \chi_{e}(\mathbf{R}, t)= & {\left[T_{N}+V_{e}(\mathbf{R})\right] \chi_{e}(\mathbf{R}, t) } \\
& +V_{d}(t) \mu_{e g}(\mathbf{R}) \chi_{g}(\mathbf{R}, t),
\end{aligned}
$$

and

$$
\begin{aligned}
i \hbar \frac{\partial}{\partial t} \chi_{k_{j} l m}(\mathbf{R}, t)= & {\left[T_{N}+V_{\text {ion }}(\mathbf{R})+\frac{\left(\hbar k_{j}\right)^{2}}{2 m_{e}}\right] \chi_{k_{j} l m}(\mathbf{R}, t) } \\
& +V_{p}(t-\Delta T) C_{l m}\left(\mathbf{R}, k_{j}, \Omega_{R}, \theta_{P}\right) \chi_{g}(\mathbf{R}, t),
\end{aligned}
$$

where $\chi_{g}, \chi_{e}$, and $\chi_{k_{j} l m}$ are wave packets on the ground $\left(V_{g}\right)$, excited $\left(V_{e}\right)$, and ion ( $\left.V_{\text {ion }}\right)$ potential energy surfaces (PES's), respectively. $T_{N}$ is the kinetic energy operator for the nuclei, $\mathbf{R}$ denotes the nuclear coordinates in a space-fixed frame (defined by the polarization directions of the laser fields), $m_{e}$ is the electron mass, and $k_{j}$ are radial quadrature points $\left(N_{k}\right.$ is the number of such points) accounting for the numerical integration over photoelectron linear momenta (angular integrations are carried out analytically by expanding the continuum wave function in spherical harmonics, $\left.Y_{l m}\right)$. $V_{d(p)}$ $=E_{d(p)} f_{d(p)}(t) \sin \left(\omega_{d(p)} t\right)$ is related to the dump $(d)$ and probe $(p)$ pulses, where $E_{d(p)}$ is the field intensity and $f_{d(p)}$ is a Gaussian envelope (time delayed for the probe pulse). Finally, $\mu_{g e}=\mu_{e g}$ is the electronic dipole transition amplitude, and the $C_{l m}$ coefficients in Eqs. (1) and (3), which have been fully discussed previously, ${ }^{11}$ provide the underlying dynamical information on the photoionization process. The molecule is oriented at angles $\Omega_{R}=\left(\phi_{R}, \theta_{R}, \gamma_{R}\right)$ with respect to the polarization vector of the dump field, and $\theta_{P}$ is the angle between the dump and probe vectors. In a compact matrix notation, Eqs. (1)-(3) may be written as

$$
\begin{aligned}
& i \hbar \frac{\partial}{\partial t} \mathbf{X}(\mathbf{R}, t)=\hat{\mathbf{H}}(\mathbf{R}, t) \mathbf{X}(\mathbf{R}, t) \\
&=\left[\hat{\mathbf{T}}(\mathbf{R})+\mathbf{V}_{D}(\mathbf{R})+\mathbf{V}_{O}(\mathbf{R})\right] \mathbf{X}(\mathbf{R}, t), \\
& \mathbf{X}(\mathbf{R}, t)=\left(\begin{array}{c}
\chi_{g}(\mathbf{R}, t) \\
\chi_{e}(\mathbf{R}, t) \\
\vdots \\
\chi_{k_{j} l m}(\mathbf{R}, t) \\
\vdots
\end{array}\right),
\end{aligned}
$$

with a similar convention for the matrix operators. $\hat{\mathbf{T}}$ is a diagonal matrix representing the kinetic energy operator and can be treated by fast Fourier transform (FFT) techniques as usual. ${ }^{55} \mathbf{V}_{D}$ is also a diagonal matrix composed of PES's for the ground, excited, and ion states. The coupling induced by the laser fields is contained in the off-diagonal matrix $\mathbf{V}_{O}$, which couples the ground state to the excited bound state as well as to the ion states. These time-dependent equations are solved using the split-operator technique ${ }^{55,56}$ and the resulting working expressions for the time evolution of nuclear wave packets are given elsewhere. ${ }^{11}$

Numerical solution of these coupled Schrödinger equations for polyatomic molecules is computationally challenging. First, it should be noted that Eq. (4) is multidimensional in two ways: the state vectors have $\left(N_{\text {ion }}+2\right)$ components, where $N_{\text {ion }}$ is the number of final ion channels $\left(k_{j}, l, m\right)$, and each such component is a multidimensional vibrational wave packet ( $\mathbf{R}$ denotes a set of vibrational modes). In particular, angular resolution of photoelectrons for large systems with low symmetry requires many $\left(k_{j}, l, m\right)$ surfaces, implying very large $N_{\text {ion. }}$. The number of vibrational modes (wavepacket dimension) as well as the number of coupled equations arising in studies of polyatomic molecules may well make the use of the FFT split-operator technique impractical. Furthermore, photoelectron amplitudes $C_{l m}$ also depend on the molecular geometry $\mathbf{R}$. For example, wave-packet propagation on $N$ mesh points with the FFT split-operator scheme, where $N$ is a composite index determined by the mesh size for all relevant vibrational coordinates, $N=N_{1} \times N_{2} \times N_{3}$ $\times \cdots$, would require that the $C_{l m}$ matrix elements be calculated $N$ times (for every point in the configuration-space mesh). Such a calculation would rapidly become prohibitive. Furthermore, if the photoionization matrix elements are expanded up to $l_{\max }$, the number of ion channels is given by $N_{\text {ion }} \simeq N_{k} \times l_{\max }^{2}$, assuming $C_{1}$ symmetry. With typical values of $N_{k}=50$ and $l_{\max }=7$, the number of ion states would be of the order of thousands, rendering such calculations impractical for a system such as chloromalonaldehyde with its 21 vibrational degrees of freedom.

It may be argued that a more efficient propagation scheme, such as the MCTDH method, ${ }^{57}$ would significantly reduce the computational effort to obtain time-resolved photoelectron spectra. In fact, full-dimensional tunneling splitting calculations were recently reported for malonaldehyde ${ }^{37}$ for both MCTDH and Monte Carlo (POITSE) schemes on a potential surface generated with the modified Shepard interpolation method. ${ }^{32}$ Such simulations on a single PES are already demanding and would be impractical for $\sim 10^{3}$ coupled potential surfaces.

\section{B. The CRS/TDH approach \\ 1. Proton-transfer dynamics on a single potential surface}

The CRS/TDH scheme has been used to simulate proton vibration dynamics in several systems, ${ }^{50-53}$ though primarily at low energies, and it would be helpful to explore its applicability to pump-probe photoelectron spectroscopic studies of proton-transfer (PT) reactions of interest here. To do so, 
we use the CRS/TDH framework described by Paramonov et $a{ }^{50}$ In this approximation the effective Hamiltonian for proton motion is given by

$$
i \hbar \frac{\partial}{\partial t} \chi(\mathbf{R}, t)=\left[T_{\mathbf{R}}+V_{0}(\mathbf{R})+V_{\mathrm{SCF}}(\mathbf{R}, t)\right] \chi(\mathbf{R}, t)
$$

with

$$
\begin{aligned}
V_{\mathrm{SCF}}(\mathbf{R}, t)= & \sum_{j=1}^{N_{\text {sub }}}\left(\frac{1}{2} \omega_{j}^{2}(\mathbf{R})\left\langle\psi_{j}\left|Q_{j}^{2}\right| \psi_{j}\right\rangle\right) \\
& +\sum_{j=1}^{N_{\text {sub }}}\left[-f_{j}(\mathbf{R})\left\langle\psi_{j}\left|Q_{j}\right| \psi_{j}\right\rangle\right. \\
& \left.+\frac{1}{2} \sum_{k \neq j=1}^{N_{\text {sub }}} K_{j k}(\mathbf{R})\left\langle\psi_{j}\left|Q_{j}\right| \psi_{j}\right\rangle\left\langle\psi_{k}\left|Q_{k}\right| \psi_{k}\right\rangle\right] .
\end{aligned}
$$

The vibrational modes have been divided in reaction $(\mathbf{R})$ and bath $\mathbf{Q}$ coordinates (in the previous section $\mathbf{R}$ represented the full set of nuclear coordinates $), \chi(\mathbf{R}, t)$ is the proton wave packet, $\psi_{j}\left(Q_{j}, t\right)$ is a bath mode wave function, and $V_{0}$ is the sudden-approximation PES for the reaction coordinates. $N_{\text {sub }}$ is the number of substrate modes, $-f_{j}$ is an element of the gradient vector (PES derivative), while $\omega_{j}^{2}$ and $K_{j k}$ are diagonal and off-diagonal elements of the Hessian matrix, respectively. We also mention in passing that the Hessian matrix has been diagonalized at the most stable molecular geometry, after projecting out rigid-body motion (the gradient has also been transformed accordingly). The time evolution of each bath mode is governed by

$$
i \hbar \frac{\partial}{\partial t} \psi_{j}\left(Q_{j}, t\right)=\left[T_{j}+\frac{1}{2} \Omega_{j}^{2}(t) Q_{j}^{2}-\mathcal{F}_{j}(t) Q_{j}\right] \psi_{j}\left(Q_{j}, t\right),
$$

where

$$
\Omega_{j}^{2}(t)=\left\langle\chi\left|\omega_{j}^{2}(\mathbf{R})\right| \chi\right\rangle
$$

and

$$
\mathcal{F}_{j}(t)=\left\langle\chi\left|f_{j}(\mathbf{R})\right| \chi\right\rangle-\frac{1}{2} \sum_{k \neq j=1}^{N_{\text {sub }}}\left\langle\chi\left|K_{j k}(\mathbf{R})\right| \chi\right\rangle\left\langle\psi_{k}\left|Q_{k}\right| \psi_{k}\right\rangle .
$$

The CRS/TDH scheme is expected to work in the vicinity of the PES minima, such as in applications to vibrations in strongly bonded systems and ground state tunneling splittings, where the harmonic approximation is reasonable. We stress that this method has been successfully applied to a number of systems, ${ }^{50-53}$ but in these studies the global (proton plus substrate) vibrational ground state was always chosen as the initial condition. For proton energies around and above the transition state (TS) barrier of the effective PES, this approximation can be expected to be poor. One generally defines the substrate normal modes by diagonalizing the Hessian matrix at the PES global minimum, and the protonsubstrate couplings are then taken into account through the first and second derivatives of the PES calculated at this point. As a result, the description of dynamical couplings becomes quite poor as the proton wave packet crosses the TS region and moves towards the other basin. For example, di- agonalization of the Hessian matrix at the planar lowestenergy structure of chloromalonaldehyde leads to 19 substrate normal vibrational modes, 13 in plane and 6 out of plane. As the proton moves towards an oxygen atom, it experiences out-of-plane forces causing the diagonal elements of the Hessian matrix $\omega_{j}^{2}(\mathbf{R})$, associated with out-of-plane modes, to be negative (imaginary $\omega_{j}$ ) in vast regions of the reaction-coordinate configuration space. This behavior is not an intrinsic problem as far as a second-order Taylor series expansion of the PES is concerned, but, according to Eq. (9), such elements define oscillator frequencies in the CRS/TDH approach. These imaginary frequencies cause the approximation to break down because the proton is eventually attracted to the region where $\omega_{j}^{2}(\mathbf{R})$ is negative (physically this region should be repulsive).

Recent applications of the CRS/MCTDH scheme ${ }^{58-60}$ have incorporated the use of flexible baths, ${ }^{49}$ in which the harmonic approximation for the substrate modes is carried out with relaxed reference geometries (the reference geometry is a function of the reaction coordinates). Though the flexible-bath approximation could in principle avoid the imaginary frequencies, out-of-plane motion can be simply neglected in the present case because estimates of the reorganization energies ${ }^{50}$ indicate that these modes are weakly coupled to PT in chloromalonaldehyde, and furthermore the average geometries are always nearly planar (see Sec. III).

\section{Optical coupling among potential surfaces}

In the MCTDH approximation, the substrate gives rise to an effective time-dependent potential acting on the proton $\left(V_{\mathrm{SCF}}\right)$, which depends on both PES derivatives and initial conditions [Eqs. (6) and (7)]. Consequently, there are no stationary vibrational levels on the different PES's and hence no well-defined resonance frequencies between electronic states, $V_{\mathrm{SCF}}^{\text {ground }}(t) \neq V_{\mathrm{SCF}}^{\text {excited }}(t)$. An attractive feature of the CRS/TDH scheme is that Eq. (8) admits an analytical solution, ${ }^{61}$ which makes determining the time evolution of bath modes in single-PES problems extremely efficient. However, the generalization of this result to studies of time-resolved photoelectron spectroscopy, where substrate modes on different PES's must be optically coupled, could pose difficulties (recall that different force fields give rise to different sets of normal modes in each PES). Treating the reaction and substrate coordinates by the same numerical approach, at least during the optical couplings, would be challenging [see Eq. (4)]. Finally, we note that even the robust MCTDH scheme would be impractical if all dynamical coordinates and coupled potential surfaces were to be taken into account, despite its efficiency in single-PES calculations. ${ }^{37,57}$

\section{CLASSICALLY AVERAGED SUBSTRATE COORDINATES}

In this section we explore a strategy well suited for addressing multidimensional wave-packet dynamics on optically coupled potential surfaces. 


\section{A. Effective potential surface of reduced dimensionality}

\section{Classically based separable potential}

Classical mechanics is often invoked in the modeling of dynamical systems with many degrees of freedom. For example, classical approximations have been used in simulations of PT in malonaldehyde ${ }^{62,63}$ and in studies of double PT in formic acid dimer. ${ }^{25}$ Sharafeddin et al. have also used a classical description of skeletal modes coupled to a proton wave packet. ${ }^{64}$ If quantum effects are not important in the substrate dynamics, this approximation would provide an efficient way to go beyond the harmonic approximation for the potential surface. However, it would not obviate the difficulties related to the optical excitation of bath modes discussed above.

Another interesting scheme is the classically based separable potential (CSP) of Gerber and co-workers ${ }^{65-68}$ which extends the applicability of the TDH approximation to considerably larger systems. The essential idea is to generate separable time-dependent single-mode potentials from classical trajectories in the following way. Suppose we have an initial Hartree-product wave function,

$$
\prod_{j} \phi_{j}\left(q_{j}\right)
$$

which is transformed into the Wigner distribution function (see below), thereby producing the initial condition for integrating a set of classical trajectories. At time $t$ the effective potential for the $i$ th mode $\bar{V}_{i}\left(q_{i}, t\right)$ is obtained by averaging over the instantaneous values of every other coordinate $\left(q_{k}\right.$ $\neq q_{i}$ ) in the corresponding set of trajectories, e.g., for the $q_{1}$ coordinate,

$$
\bar{V}_{1}\left(q_{1}, t\right)=\frac{1}{N} \sum_{\alpha=1}^{N} V\left(q_{1}, q_{2}^{\alpha}(t), q_{3}^{\alpha}(t), \ldots\right) W^{\alpha},
$$

where $\alpha$ runs over the set of trajectories and $W^{\alpha}$ is a weighting factor arising from the initial condition. In this way, one bypasses the most time-consuming step in TDH simulations, namely, the self-consistent calculation of intermode couplings, which are now described solely through CSP potentials.

\section{Averaging over the skeleton geometry}

Although the CSP method is useful within the TDH scheme, time-dependent effective potentials are still invoked. We now carry the idea of generating an average potential from classical trajectories a step further to develop an approach that is well suited for application to time-resolved photoelectron spectroscopy studies. The averaging over ensembles of classical trajectories and its connection to the CSP scheme may be derived from the classical limit of a Wigner distribution function. Suppose we have a general wave function for a nuclear wave packet,

$$
\varphi(\mathbf{x}, t)
$$

where the vector $\mathbf{x}$ stands for all the coordinates collectively. The corresponding Wigner phase-space distribution function can be constructed as

$$
\begin{aligned}
\Gamma_{\varphi}(\mathbf{x}, \mathbf{p}, t)= & (\pi \hbar)^{-N} \int d y \varphi^{*}(\mathbf{x}-\mathbf{y}, t) \varphi(\mathbf{x}+\mathbf{y}, t) \\
& \times \exp [2 i \mathbf{p} \cdot \mathbf{y} / \hbar],
\end{aligned}
$$

where $\Gamma_{\varphi}(\mathbf{x}, \mathbf{p}, t)$ satisfies the classical Liouville equation

$$
\left(\frac{\partial}{\partial t}+\frac{\partial H}{\partial \mathbf{p}} \cdot \frac{\partial}{\partial \mathbf{x}}-\frac{\partial H}{\partial \mathbf{x}} \cdot \frac{\partial}{\partial \mathbf{p}}\right) \Gamma_{\varphi}(\mathbf{x}, \mathbf{p}, t)=0
$$

as $\hbar \rightarrow 0$. It is instructive to rewrite $\Gamma_{\varphi}(\mathbf{x}, \mathbf{p}, t)$ to distinguish the reaction coordinates $(\mathbf{R})$ from those of the bath $(\mathbf{Q})$, $\mathbf{x}=(\mathbf{R}, \mathbf{Q})$. The associated momenta may also be written as $\mathbf{p}=(\mathbf{P}, \boldsymbol{\Pi})$ such that

$$
\Gamma_{\varphi}(\mathbf{x}, \mathbf{p}, t) \equiv \Gamma_{\varphi}(\mathbf{R}, \mathbf{P}, \mathbf{Q}, \boldsymbol{\Pi}, t) .
$$

The semiclassical Wigner function can therefore be propagated in terms of classical trajectories $\left(\mathbf{R}_{i}(t), \mathbf{P}_{i}(t), \mathbf{Q}_{i}(t), \boldsymbol{\Pi}_{i}(t)\right)$ with the suffix $i$ labeling the trajectories,

$$
\Gamma_{\varphi}\left(\mathbf{R}_{i}(t), \mathbf{P}_{i}(t), \mathbf{Q}_{i}(t), \boldsymbol{\Pi}_{i}(t)\right) .
$$

We now divide the reaction configuration space $\mathbf{R}$ into several small regions denoted by the index $a$, and $\mathbf{R}_{i}^{(a)}(t)$ denotes the $i$ th trajectory with a component in the $a$ th region. Whenever trajectories cross this region, the bath coordinates $\mathbf{Q}$ may be averaged as

$\left\langle\mathbf{Q}\left(\mathbf{R}^{(a)}, t\right)\right\rangle_{\varphi}=\sum_{i} \Gamma_{\varphi}\left(\mathbf{R}_{i}^{(a)}(t), \mathbf{P}_{i}^{(a)}(t), \mathbf{Q}_{i}(t), \boldsymbol{\Pi}_{i}(t)\right) \mathbf{Q}_{i}(t)$.

It is thus reasonable, though approximate, to regard the reduced reaction-coordinate wave packet,

$$
\bar{\Gamma}_{\varphi}(\mathbf{R}, \mathbf{P}, t) \equiv \int \Gamma_{\varphi}(\mathbf{R}, \mathbf{P}, \mathbf{Q}, \Pi, t) d \mathbf{Q} d \mathbf{\Pi},
$$

as being propagated on the effective time-dependent potential,

$$
V\left(\mathbf{R}^{(a)},\left\langle\mathbf{Q}\left(\mathbf{R}^{(a)}, t\right)\right\rangle_{\varphi}, t\right),
$$

where the average skeleton positions are placed at $\left\langle\mathbf{Q}\left(\mathbf{R}^{(a)}, t\right)\right\rangle_{\varphi}$, whenever the reaction coordinates are found in region $a, \mathbf{R}^{(a)}$. Propagating the reduced wave packet $\varphi\left(\mathbf{R}^{(a)}, t\right)$ in this fashion on the above effective potential is clearly similar to the CSP framework.

\section{A time-independent potential}

The effective potential of Eq. (20) depends both on time and on the initial conditions for the wave packet, just as in the CSP approach. For the present application it would be advantageous to further remove the time dependence from the potential. This may be achieved by averaging $\left\langle\mathbf{Q}\left(\mathbf{R}^{(a)}, t\right)\right\rangle_{\varphi}$ over time, 


$$
\left\langle\mathbf{Q}\left(\mathbf{R}^{(a)}\right)\right\rangle_{\varphi}=\frac{1}{T} \int_{0}^{T}\left\langle\mathbf{Q}\left(\mathbf{R}^{(a)}, t\right)\right\rangle_{\varphi} d t,
$$

and over initial conditions. This averaging of substrate coordinates, denoted by double brackets,

$$
\left\langle\left\langle\mathbf{Q}\left(\mathbf{R}^{(a)}\right)\right\rangle\right\rangle=\int d \varphi\left\langle\mathbf{Q}\left(\mathbf{R}^{(a)}\right)\right\rangle_{\varphi},
$$

results in an effective potential for the reaction coordinates that depends neither on time nor on the choice of the initial reduced wave packet, a convenient feature for the application of interest here since pumped (dumped) wave packets have arbitrary shapes. Consider, for example, a system with $(N+2)$ atomic coordinates, namely, two reaction coordinates ( $x, y$ proton coordinates) and $N$ substrate coordinates, and a set of $M$ trajectories. The reaction-coordinate configuration space may then be arbitrarily divided according to $x_{1}<x_{2}$ $<x_{3}<\cdots$ and $y_{1}<y_{2}<y_{3}<\cdots$. Let us also assume that PT takes place along the $O x$ direction and define the segments $s_{i j}=\left(x_{i}, y_{j} \leqslant y<y_{j+1}\right)$. For every $s_{i j}$ we may examine the entire set of trajectories and record all the times when the reaction coordinates are found in each segment. If the reaction coordinates cross the $s_{i j}$ segment $n_{i j}^{\alpha}$ times in the $\alpha$ th trajectory, the total number of crossings in this particular segment $\left(N_{i j}\right)$ is obtained by summing over the trajectories,

$$
N_{i j}=\sum_{\alpha=1}^{M} n_{i j}^{\alpha} .
$$

We now denote by $q_{r}^{\alpha \nu}\left(s_{i j}\right)$ the value of the $r$ th skeleton coordinate when the reaction coordinates cross $s_{i j}$ for the $\nu$ th time in the $\alpha$ th trajectory. An average value for each substrate coordinate may be obtained as

$$
\bar{q}_{r}\left(s_{i j}\right)=\frac{1}{N_{i j}} \sum_{\alpha=1}^{M} \sum_{\nu=1}^{n_{i j}^{\alpha}} q_{r}^{\alpha \nu}\left(s_{i j}\right) .
$$

Hence, the vector $\left(\bar{q}_{1}\left(s_{i j}\right), \bar{q}_{2}\left(s_{i j}\right), \ldots, \bar{q}_{N}\left(s_{i j}\right)\right)$ could be viewed as representing the average skeleton geometry when the proton is found in the $s_{i j}$ segment [note that Eq. (24) is an approximation to Eq. (22)]. We could also consider points $\left(x_{i}, y_{j}\right)$ instead of segments, but the crossing of such points by individual trajectories would be extremely unlikely.

The ideas underlying such classically averaged potential surfaces may be summarized by considering the effective Hamiltonian,

$$
H=T_{\mathbf{R}}+V_{Q}(\mathbf{R}),
$$

where $\bar{Q}(\mathbf{R}) \equiv\left\{\bar{q}_{1}(\mathbf{R}), \ldots, \bar{q}_{N}(\mathbf{R})\right\}$. The effective potential $V_{\bar{Q}}^{-}$ arising from the classical bath averaging of Eq. (22) incorporates skeleton relaxation in an average sense. It thus provides a model of reduced dimensionality that takes into account only the reaction coordinates $(\mathbf{R})$ and is suitable for studies of time-resolved photoelectron spectra. Equation (25) omits kinetic energy terms involving the bath modes. Inclusion of these terms ${ }^{69}$ would be necessary to obtain quantitative results, but here we seek only signatures of PT in timeresolved photoelectron spectra and therefore prefer the simplicity of Eq. (25). We expect our model to describe cor- rectly the qualitative changes in photoelectron signals arising from proton motion across the barrier, although the resulting PT periods or dwell time between successive transfers might not be realistic. With its time-independent and parameter-free potentials, embodying multidimensional effects in an average sense, and a convenient framework for incorporating $a b$ initio photoionization matrix elements, the present model possesses advantages over the harmonic potentials employed elsewhere ${ }^{48}$ without significantly increasing the computational complexity of studies of time-resolved photoelectron spectra.

Classically averaged potentials should by no means be restricted to proton-transfer reactions, but should be broadly applicable to any dynamical process where reaction and skeleton modes can be meaningfully distinguished. It could be viewed as a general approach to obtaining effective reaction energy surfaces of reduced dimensionality, where the computational effort is determined by the quantum-chemical method employed to obtain the effective potential surfaces and by the number of vibrational coordinates.

These classically averaged potential surfaces bring about significant simplification in studies of time-resolved photoelectron spectra. They provide an efficient approach to obtaining time-independent reaction surfaces of reduced dimensionality which allow for proton-substrate coupling in an average sense and avoid difficulties associated with optical excitation of bath modes, without invoking approximations as unrealistic as frozen or adiabatic skeletons. To our knowledge, the only $a b$ initio calculation of time-resolved photoelectron cross sections reported for a polyatomic system ${ }^{16}$ included only two vibrational modes. While it is true that further inclusion of multidimensional couplings would eventually become prohibitive, the complete neglect of other vibrational coordinates can be overly restrictive. In the present application, for example, time-resolved photoelectron spectra for intramolecular PT in chloromalonaldehyde would be simulated by generating ab initio photoionization matrix elements over a two-dimensional reaction grid for the proton, $\mathbf{R}=(x, y)$, but for a skeleton geometry that changes in accordance with $\overline{\mathbf{Q}}(\mathbf{R})$, thereby introducing multidimensional effects in an average sense into the matrix elements without a concomitant increase in computational effort.

\section{B. Generation of classically averaged potential surfaces}

We illustrate the proposed procedure for obtaining classically averaged potential surfaces with an application to PT in chloromalonaldehyde. In these simulations we employ quantum-chemical potential surfaces obtained at the restricted Hartree-Fock (RHF) level with the 6-31G $(d, p)$ basis internal to the GAMESS package. ${ }^{70}$ The classical equations of motion were integrated using the locally analytic integrator (LAI) with an integration step of $0.3 \mathrm{fs}$. LAI is known to be an efficient integrator for classical and semiclassical calculations in molecular systems. ${ }^{71}$ The initial position of each atom was randomly sampled about their values at the global minimum, keeping $\left|\mathbf{R}_{\text {ini }}-\mathbf{R}_{\text {opt }}\right|<0.3 \AA$. Two hundred such initial positions were chosen and the related trajectories 


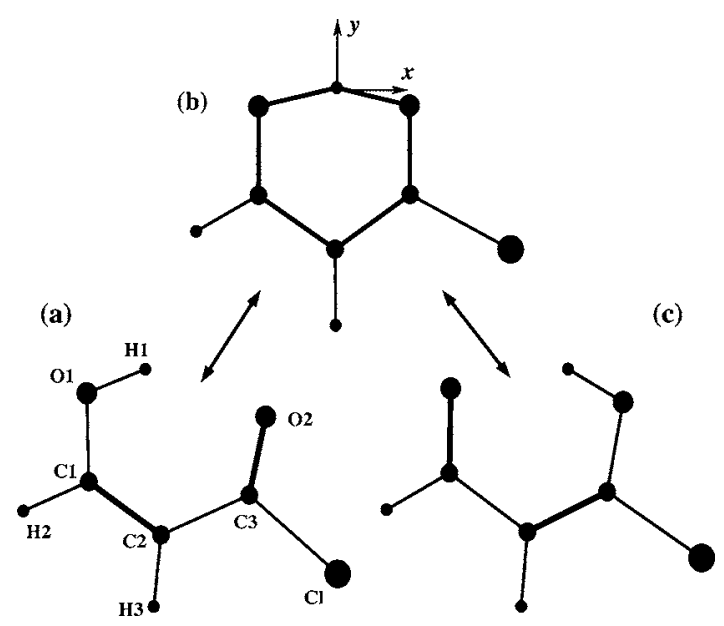

FIG. 2. Intramolecular proton transfer in chloromalonaldehyde. Structure (a) is the most stable (global minimum), (b) is the transition state, and (c) is the secondary minimum. The frame of reference for the reaction coordinates is indicated in structure (b).

evolved for 15 fs. The total translational and angular momenta were then reset to zero and the magnitudes of the internal momenta rescaled to obtain the following total energies: $0.52,0.79,1.06,1.34, \ldots, 4.06 \mathrm{eV}$ (the optimized RHF transition state barrier is $0.65 \mathrm{eV}$ ). The resulting atomic positions and momenta were taken as initial conditions for the classical dynamical calculations and each trajectory was evolved for $150 \mathrm{fs}$ which is long enough for PT to occur. Of 2800 trajectories, 215 showed at least one PT and only this subset was considered in the averaging process of Eq. (24) (only trajectories with energies above $1.6 \mathrm{eV}$ showed PT). The criterion for PT was based on the value of the dimensionless coordinate $\eta=R_{\mathrm{O}_{1} \mathrm{H}_{1}} \cos \theta / R_{\mathrm{O}_{1} \mathrm{O}_{2}}$ at the RHF transition state geometry, where $\theta$ is the angle between $\mathrm{O}_{1}-\mathrm{H}_{1}$ and $\mathrm{O}_{1}-\mathrm{O}_{2}$ bonds (see Fig. 2 for atomic labels). Since averaging may introduce overall rotations and translations, rigid-body motion was projected out of the average geometry using the optimal configuration as reference for the displacements, $\delta \mathbf{R}=\left(\mathbf{R}_{\text {ave }}-\mathbf{R}_{\text {opt }}\right)$. Throughout this paper, the reactioncoordinate frame is chosen as indicated in Fig. 2, with the origin located on the proton in the optimal RHF transition state [structure (b)] and with the $O x$ and $O y$ axes pointing rightward and upward, respectively. The global minimum (left basin) is located at $x<0$, and the secondary minimum (right basin) at $x>0$.

The resulting average coordinates as a function of the reaction coordinates $(x, y)$ are shown in Fig. 3 for $\mathrm{O}_{1}$ and $\mathrm{O}_{2}$, the skeleton atoms undergoing largest displacements. The values of the $y$ reaction coordinate are taken at midpoints of the $s_{i j}$ segments. The average values (filled symbols) are not very smooth because the number of trajectories is not large enough, and the dashed lines with hollow symbols show polynomial fits to the average values. Though not shown here, the average displacements along the $\mathrm{Oz}$ direction (outof-plane) were very small $(<0.01 \AA)$ for all atoms. Furthermore, because the dependence of these averaged coordinates on the $y$ reaction coordinate was quite small, we also show the average of these polynomial regressions for different $y$ values (thick solid lines). This amounts to viewing the aver-
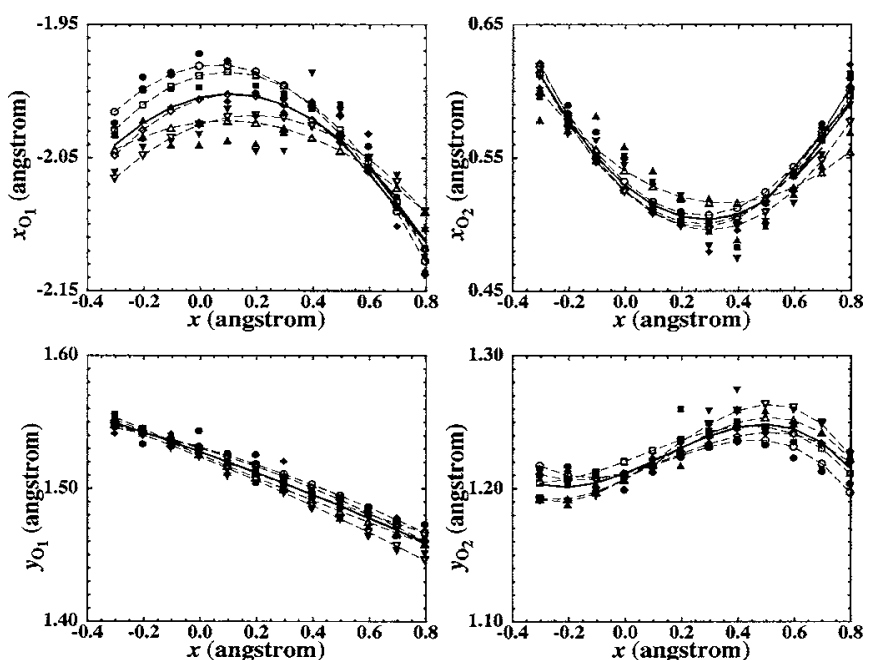

FIG. 3. Average values of selected skeleton coordinates $\left(x_{\mathrm{O}_{1}}, y_{\mathrm{O}_{1}}, x_{\mathrm{O}_{2}}, y_{\mathrm{O}_{2}}\right)$ for $\mathrm{O}_{1}$ and $\mathrm{O}_{2}$ (Fig. 2) as functions of the reaction coordinates $(x, y)$. The filled symbols are averages over classical trajectories, while the dashed lines with hollow symbols are the corresponding polynomial regressions. The thick solid lines are $y$ averages over the regression polynomials. Circles: $y=-0.136 \AA$; squares: $y=-0.086 \AA$; diamonds: $y=-0.036 \AA$; up triangles: $y=0.014 \AA$; down triangles: $y=0.064 \AA$.

age geometry as a function of only the $x$ reaction coordinate. Though it enclosed the two minima and the TS, the reactioncoordinate configuration space containing all the $s_{i j}$ segments with at least one crossing was quite small, especially along the $O y$ direction. Outside this region, the skeleton coordinates were frozen at the boundary values, $q_{r}\left(x<x_{\min }\right)$ $=q_{r}\left(x_{\min }\right)$ and $q_{r}\left(x>x_{\max }\right)=q_{r}\left(x_{\max }\right)$. The average geometries were obtained with regression polynomials of degrees 2 and 3 , depending on the particular behavior of skeleton coordinates (the $z$ coordinates were adjusted with straight lines). The global minimum of the resulting averaged PES was found to lie $0.044 \mathrm{eV}$ above the optimized global minimum (lowest-energy configuration). A different choice for the polynomial fit (all of degree 2 for $x, y$ skeleton coordinates) leads to essentially the same PES $\left(\sim 10^{-2} \mathrm{eV}\right.$ differences at the stationary structures).

In Fig. 4 we compare the ground state potential surfaces obtained with the three relevant approximations for the molecular skeleton, namely, sudden (frozen bath), classically averaged, and adiabatic (fully relaxed bath). In the right panel, second-order Møller-Plesset (MP2) energies were added to the potential surfaces shown in the left panel (classical simulations on the MP2 surface were not carried out). The classically averaged potential surfaces (central panel) are seen to be intermediate between the frozen (upper panel) and adiabatic (lower panel) ones. The energies of the stationary structures (TS and right basin minimum) are shown in Table I, where MP2 calculations are also seen to yield very low barriers. The frozen-geometry approximation leads to highly asymmetric potentials, with a right basin that is rather small and extremely shallow (see Table I). On the other hand, the adiabatic (relaxed) skeleton approximation yields shallow and very wide right basins, thus overestimating the asymmetry in the opposite direction. Neither of these two extreme approximations would seem to provide a reasonable description of PT dynamics. 

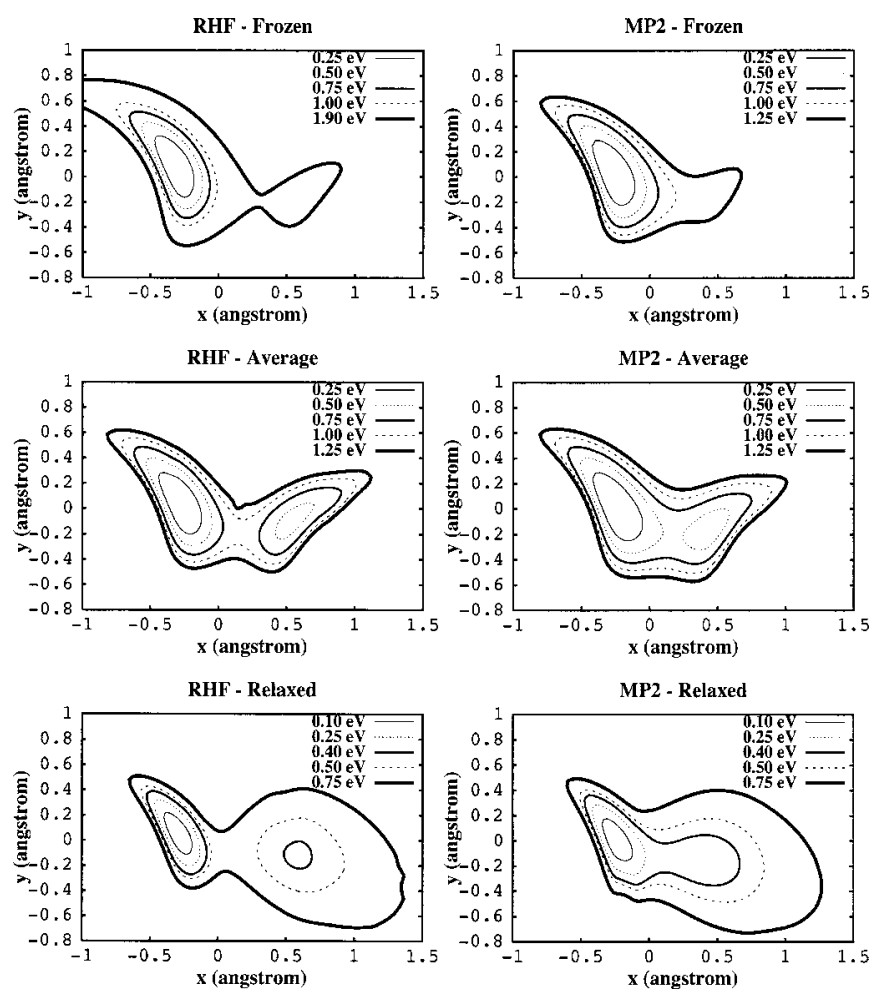

FIG. 4. Potential energy surfaces for the electronic ground state of chloromalonaldehyde. Plots on the left column were calculated at the RHF level, while those on the right at the MP2 level. Upper panel: sudden (frozen) approximation; central panel: average geometry; lower panel: fully relaxed (adiabatic) approximation.

The influence of electron correlation on the potential surface for the classically averaged skeleton geometry is also illustrated in Table I, where the energies of the stationary structures are given in different approximations, namely, RHF, MP2, and configuration interaction with single and double excitation (CISD). We assumed average geometries obtained from trajectories on the RHF potentials since the use of a higher-level description of the surface in the classical simulations would be extremely time consuming. MP2 calculations often underestimate the TS barrier due to the lack of variational stability.

A few relevant geometrical parameters (interatomic dis-

TABLE I. A $b$ initio total energy at the stationary structures of chloromalonaldehyde. Transition state (TS) and secondary minimum (SM) heights are given in units of $\mathrm{eV}$ with respect to the respective global minima. Ground state potential surfaces were calculated at the RHF, MP2, and CISD levels, with substrate modes treated in the sudden (frozen), classically averaged (average), and adiabatic (fully relaxed) approximations. MP2 and CISD classically averaged results were obtained by adding the respective correlation energies to the RHF-averaged geometries, while sudden- and adiabaticapproximation optimizations were carried out with the appropriate correlated potentials.

\begin{tabular}{lccccccccc}
\hline \hline & \multicolumn{2}{c}{ RHF } & & \multicolumn{2}{c}{ MP2 } & & \multicolumn{2}{c}{ CISD } \\
\cline { 2 - 3 } \cline { 8 - 9 } \cline { 8 - 9 } & & TS & SM & & TS & SM & & TS & SM \\
\hline Frozen & 1.868 & 1.514 & & 1.060 & 1.027 & & 1.459 & 1.299 \\
Average & 0.936 & 0.407 & & 0.533 & 0.332 & & 0.755 & 0.390 \\
Relaxed & 0.651 & 0.383 & & 0.363 & 0.335 & & 0.491 & 0.355 \\
\hline \hline
\end{tabular}

tances and angles) for the stable structures of chloromalonaldehyde for the averaged and optimal geometries are given in Table II. Though the classically averaged minima are shifted towards the geometry of the tautomer in comparison with the optimal geometries (see the $\mathrm{O}_{1}-\mathrm{O}_{2}, \mathrm{C}_{1}-\mathrm{C}_{2}, \mathrm{C}_{3}-\mathrm{O}_{2}, \mathrm{O}_{1}-\mathrm{C}_{1}$, and $\mathrm{C}_{2}-\mathrm{C}_{3}$ distances), the most striking difference arises at the transition state, where the classically averaged structure gives rise to larger $\mathrm{H}_{1}-\mathrm{O}_{1}$ and $\mathrm{H}_{1}-\mathrm{O}_{2}$ distances. Finally, we note that a Mulliken population analysis of the results for the averaged geometry clearly manifests the change in electronic structure associated with tautomerization. Classical trajectories often indicate that proton-transfer events and tautomerization occur essentially on the same time scale, ${ }^{25}$ as illustrated in Fig. 5, where the circles indicate time-averaged bond lengths before and after PT, i.e., averages for $0 \leqslant t$ $\leqslant t_{\mathrm{PT}}$ and $t_{\mathrm{PT}} \leqslant t \leqslant t_{\text {final }}$, respectively.

\section{Comparison between classically averaged geometry and CRS/TDH dynamics}

Classically averaged surfaces and the CRS/TDH approximation describe proton-substrate couplings in different ways. Though the former can be expected to adequately describe these couplings at energies around the TS energy and the latter to do so only at the bottom of the potential surface, the comparison of these approaches through numerical simulations could be insightful. Such a comparison may be carried out by taking advantage of appropriate time averages in CRS/TDH dynamics, since time-independent couplings are embedded in the average skeleton geometries. Though any differences should be more evident at higher energies, we confine our comparison to the vicinity of global minima where the CRS/TDH framework is expected to work well. The CRS/TDH dynamical simulations were carried out using sudden-approximation potentials calculated at the RHF and MP2 levels (see Fig. 4) as reference geometries. Since outof-plane skeleton vibrations are weakly coupled to proton motion, only the 13 in-plane bath modes were taken into account. For each of these modes, the initial condition was assumed to be the ground state of a driven harmonic oscillator with frequency and force given by Eqs. (9) and (10) at the initial time [note that the intermode coupling term in Eq. (10) requires a self-consistent procedure], and the initial proton wave packet was assumed to be the vibrational ground state of the sudden-approximation PES. With this low-energy initial condition, the wave packets showed only small oscillations with little spreading over 2 ps.

We obtained time averages of the expectation values for several geometrical parameters (distances and angles) of the molecular skeleton. Table III compares these parameters with those of the optimal geometry and the global minimum geometry of the classically averaged PES. At the RHF level, the classically averaged values are shifted towards the transition state geometry (shorter $\mathrm{O}_{1}-\mathrm{O}_{2}$ distance, longer $\mathrm{C}_{1}-\mathrm{C}_{2}$ and $\mathrm{C}_{3}-\mathrm{O}_{2}$ bonds, and shorter $\mathrm{O}_{1}-\mathrm{C}_{1}$ and $\mathrm{C}_{2}-\mathrm{C}_{3}$ bonds), but remain close to the values at the optimal geometry. The CRS/TDH time averages obtained at the RHF level are even closer to the optimal geometry and show no tendency towards tautomerization. This difference between the classically averaged and the CRS/TDH time-averaged values may 
TABLE II. Geometrical parameters of the stable structures. Angles are given in degrees and distances in $\AA$. We compare results from full geometry optimizations (optimized) with those from averaging over classical trajectories (average). All results were obtained at the RHF level with a 6-31G $(d, p)$ basis set.

\begin{tabular}{ccccccccc}
\hline \hline & \multicolumn{2}{c}{ Global minimum } & & \multicolumn{2}{c}{ Transition state } & & \multicolumn{2}{c}{ Secondary minimum } \\
\cline { 2 - 3 } Parameter & Optimized & Average & & Optimized & Average & & Optimized & Average \\
\hline $\mathrm{H}_{1}-\mathrm{O}_{1}$ & 0.9533 & 0.9633 & & 1.2324 & 1.3190 & & 1.8045 & 1.7456 \\
$\mathrm{H}_{1}-\mathrm{O}_{2}$ & 1.9351 & 1.8397 & & 1.1493 & 1.2633 & & 0.9624 & 0.9592 \\
$\mathrm{O}_{1}-\mathrm{C}_{1}$ & 1.3093 & 1.3046 & & 1.2472 & 1.2722 & & 1.2075 & 1.2127 \\
$\mathrm{C}_{1}-\mathrm{C}_{2}$ & 1.3426 & 1.3592 & & 1.4018 & 1.3967 & & 1.4516 & 1.4469 \\
$\mathrm{C}_{2}-\mathrm{C}_{3}$ & 1.4496 & 1.4493 & & 1.3839 & 1.4104 & & 1.3429 & 1.3615 \\
$\mathrm{C}_{3}-\mathrm{O}_{2}$ & 1.1865 & 1.2096 & & 1.2492 & 1.2430 & & 1.3005 & 1.3197 \\
$\mathrm{O}_{1}-\mathrm{O}_{2}$ & 2.7099 & 2.6577 & & 2.3261 & 2.5273 & & 2.6288 & 2.5780 \\
$\mathrm{O}_{1}-\mathrm{H}_{1}-\mathrm{O}_{2}$ & 136.82 & 140.92 & & 155.17 & 156.31 & & 141.76 & 143.14 \\
\hline \hline
\end{tabular}

arise from the contribution of higher-energy trajectories (comparable to TS and above) to classical averages, since vibrationally excited states of strongly coupled modes can be expected to play a major role in the PT process. We also note that employing an initial wave packet with equal weights for the ground and first excited vibrational states did not significantly affect the time averages (further inclusion of higher excited states in the CRS/TDH scheme would be question-
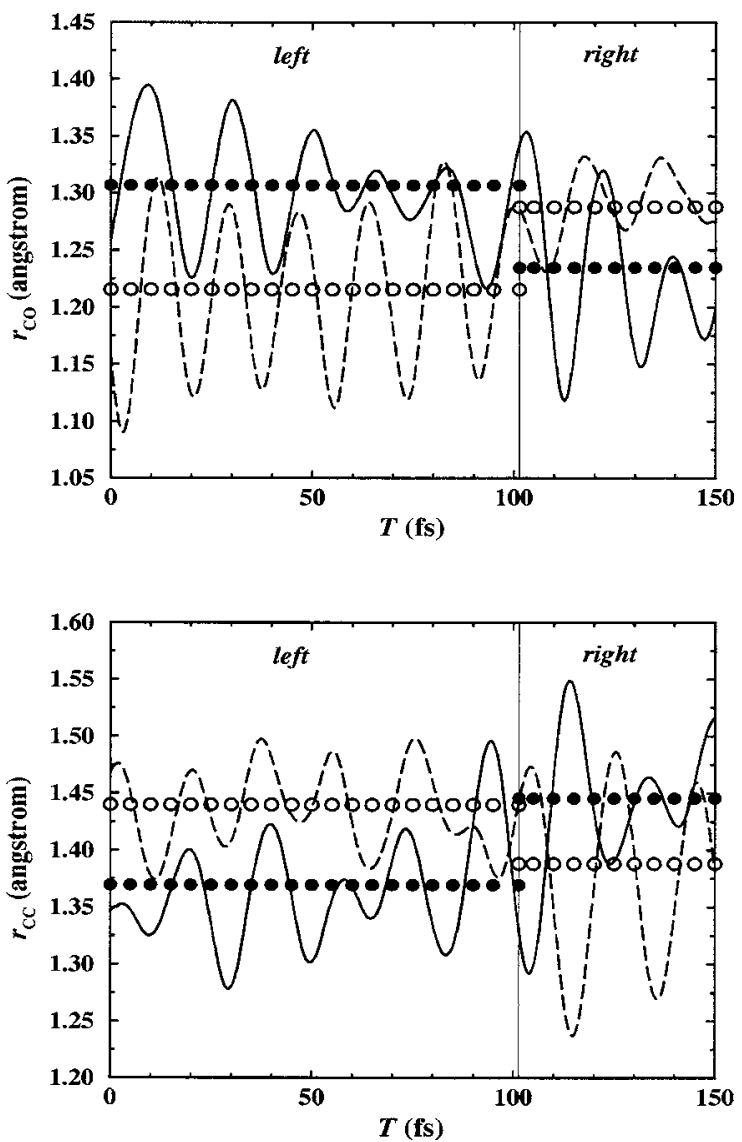

FIG. 5. Time evolution of $\mathrm{C}-\mathrm{O}$ (upper panel) and $\mathrm{C}-\mathrm{C}$ (lower panel) bond lengths in a typical trajectory. The vertical lines around $100 \mathrm{fs}$ indicate the proton-transfer time from the global minimum basin (left) to the secondary minimum basin (right). Upper panel: solid line: $\mathrm{C}_{1}-\mathrm{O}_{1}$; dashed line: $\mathrm{C}_{3}-\mathrm{O}_{2}$ (see Fig. 2 for labels). Lower panel: solid line: $\mathrm{C}_{1}-\mathrm{C}_{2}$; dashed line: $\mathrm{C}_{2}-\mathrm{C}_{3}$. In both panels, the circles indicate time-averaged bond lengths before and after proton transfer. able). At the MP2 level, the time-averaged geometry is also close to the reference geometry with no noticeable signature of tautomerization, and the MP2 classically averaged values are essentially the same as those obtained at the RHF level. This could be expected since we simply added MP2 correlation energies to the RHF-averaged potentials, i.e., we did not employ MP2 potentials in generating the classical trajectories, because generating classical trajectories with higherlevel potentials would be much more time consuming.

The results in Table III also show the skeleton parameters with the largest amplitudes in the CRS/TDH simulations to be the $\mathrm{O}_{1}-\mathrm{C}_{1}$ and $\mathrm{O}_{1}-\mathrm{O}_{2}$ distances, suggesting significant coupling of oxygen atoms to proton motion. Though this is the case in both the RHF and MP2 simulations, the potential derivatives on the RHF and MP2 surfaces were rather different, and, as a result, there is no clear one-to-one correspondence between the respective normal modes. Further insight into the strength of the dynamical protonsubstrate couplings may be gained from the weighted reorganization energy,

$$
\Delta \bar{\varepsilon}=\sum_{j} \int d \mathbf{R} \Delta \varepsilon_{j}(\mathbf{R})|\chi(\mathbf{R})|^{2},
$$

where $\chi$ is the proton wave function and $\Delta \varepsilon_{j}$ the reorganization energy of the $j$ th bath mode. ${ }^{50}$ Because of the lowenergy initial condition, the proton wave packet can be written as

$$
\chi(\mathbf{R}, t) \simeq c_{0}(t) \phi_{0}(\mathbf{R})+c_{1}(t) \phi_{1}(\mathbf{R}),
$$

with $\phi_{0,1}$ being the ground and first excited vibrational eigenstates of the frozen-bath potential surface. The reorganization energies averaged over the ground and first excited vibrational eigenstates are, respectively, 2.63 and $3.80 \mathrm{eV}$ for the MP2 potential, and 3.09 and $4.25 \mathrm{eV}$ for the RHF potential. Though these values suggest somewhat stronger couplings on the RHF surface, the oscillation amplitudes in Table III indicate more effective couplings at the MP2 level. This may be understood by noting that the steeper left basin of the RHF potential (see Fig. 4) hinders proton motion. The MP2 potential shows a smaller slope towards the right basin, thus allowing stronger couplings as the proton reaches a more distant turning point. 
TABLE III. Expectation values of selected geometrical parameters of the molecular skeleton. Angles are given in degrees and distances in $\AA$. The Reference column lists the results from ab initio geometry optimization (global minimum), and results in the Time average column were obtained from 2 ps simulations using the $\mathrm{CRS} / \mathrm{TDH}$ scheme (root-mean-square deviations in parentheses). The Classical average column lists the global minimum geometry of the classically averaged PES.

\begin{tabular}{cccccccc}
\hline \hline & \multicolumn{3}{c}{ RHF level } & & \multicolumn{3}{c}{ MP2 level } \\
\cline { 2 - 3 } Parameter & Reference & $\begin{array}{c}\text { Time } \\
\text { average }\end{array}$ & $\begin{array}{c}\text { Classically } \\
\text { averaged }\end{array}$ & & Reference & $\begin{array}{c}\text { Time } \\
\text { average }\end{array}$ & $\begin{array}{c}\text { Classically } \\
\text { averaged }\end{array}$ \\
\hline $\mathrm{O}_{1}-\mathrm{C}_{1}$ & 1.3093 & $1.3235(0.0059)$ & 1.3046 & & 1.3306 & $1.3553(0.0101)$ & 1.3052 \\
$\mathrm{C}_{1}-\mathrm{C}_{2}$ & 1.3426 & $1.3391(0.0018)$ & 1.3592 & & 1.3612 & $1.3550(0.0031)$ & 1.3600 \\
$\mathrm{C}_{2}-\mathrm{C}_{3}$ & 1.4496 & $1.4491(0.0036)$ & 1.4493 & & 1.4410 & $1.4416(0.0058)$ & 1.4481 \\
$\mathrm{C}_{3}-\mathrm{O}_{2}$ & 1.1865 & $1.1837(0.0037)$ & 1.2096 & & 1.2251 & $1.2156(0.0079)$ & 1.2056 \\
$\mathrm{O}_{1}-\mathrm{O}_{2}$ & 2.7099 & $2.7088(0.0094)$ & 2.6577 & & 2.6532 & $2.6597(0.0164)$ & 2.6433 \\
$\mathrm{C}_{1}-\mathrm{C}_{2}-\mathrm{C}_{3}$ & 119.85 & $119.50(0.36)$ & 118.54 & & 118.96 & $118.68(0.55)$ & 118.35 \\
\hline \hline
\end{tabular}

\section{APPLICATION TO DUMP-PROBE PHOTOELECTRON SPECTROSCOPY}

\section{A. Dump-probe scheme}

Our goal is to employ these potential energy surfaces of reduced dimensionality in simulations of time-resolved dump-probe photoelectron spectra for proton transfer in chloromalonaldehyde. We recently explored the potential of such time-resolved spectra for tracking the proton dynamics in chloromalonaldehyde using a model two-dimensional PES and model photoionization amplitudes. ${ }^{48}$ These studies suggested that it would be feasible to obtain a fairly localized wave packet on the ground state PES by dumping a wave packet initially delocalized on the excited state PES. Moreover, the photoelectron signals were seen to probe the passage of the wave packet across the transition state region on the ground state PES. We now revisit these model studies to show that these important conclusions also hold with the classically averaged PES. We consider the dump-probe scheme of Fig. 1. A wave packet on the excited state PES is dumped to the electronic ground state by a linearly polarized ultrashort pulse of frequency $\omega_{d}$, and is subsequently ionized by a linearly polarized probe pulse of frequency $\omega_{p}$ after a time delay $\Delta T$. The formulation of time-resolved dumpprobe photoelectron spectroscopy employed here has been briefly outlined in Sec. II. As discussed in Refs. 11 and 48, the total photoelectron signals are given by

$$
P_{\text {ion }}=\sum_{l, m} \int d \mathbf{R} \int d k k^{2}\left|\chi_{k l m}(\mathbf{R})\right|^{2},
$$

where $\chi_{k l m}$ is the ion wave packet [see Eqs. (1) and (3)]. To explore how the photoelectron signals may monitor the PT wave-packet dynamics on the ground state we assume a constant photoionization matrix element throughout. Results employing $a b$ initio values of these matrix elements will be discussed elsewhere. ${ }^{72}$

\section{B. Vertical approximation for ionization}

The ground state, excited state, and ion ground state PES's employed in this dump-probe photoelectron spectroscopic study of proton transfer in chloromalonaldehyde are shown in Fig. 6. We assume that all optical excitations are vertical and because we are probing PT on the ground state, all PES's were obtained at the classically averaged geometry of the ground state. The PES's for the ground and ion states were determined at the HF level, while the PES for the excited state $\left(2{ }^{1} A^{\prime}\right)$ was obtained from a single-excitation CI (CIS) calculation. The $2 A^{\prime}$ state lies slightly above the first
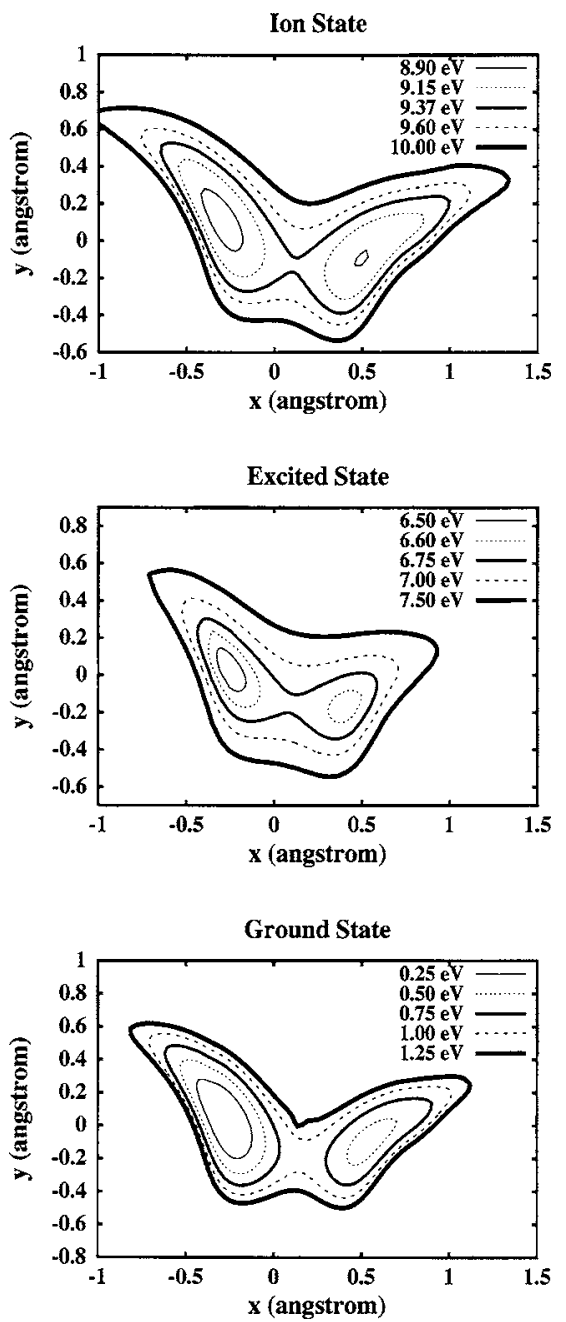

FIG. 6. Potential energy surfaces for the electronic ground state (lower panel), excited state (central panel), and ion state (upper panel) of chloromalonaldehyde. All potential surfaces were calculated at the classically averaged geometry of the ground state. 

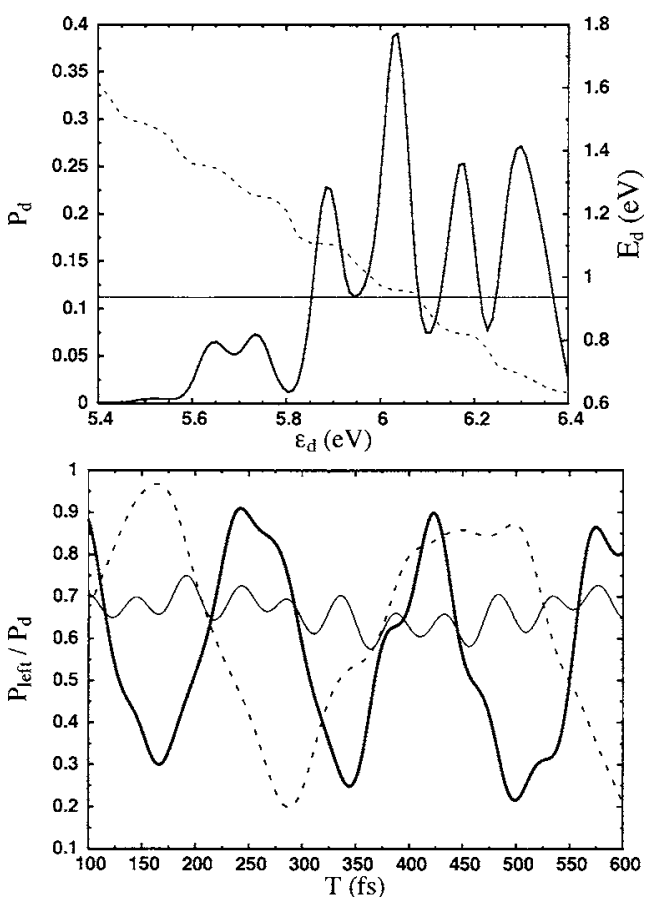

FIG. 7. Upper panel: dump energy $\left(\varepsilon_{d}=\hbar \omega_{d}\right)$ dependence of the ground state population, $P_{d}$ (solid line, scales to the left). Also shown is the dependence of the final wave-packet energy, $E_{d}$ (dashed line, scales to the right). The horizontal line indicates the ground state proton-transfer barrier. Lower panel: time evolution of the left-population ratio for selected dump energies. Dashed line: $5.75 \mathrm{eV}$; thick solid line: $5.95 \mathrm{eV}$; thin solid line: $6.10 \mathrm{eV}$.

excited singlet state $\left(1^{1} A^{\prime}\right)$ but has a large transition dipole moment with the ground electronic state. The wave packet on the ground state surface may be formed by dumping a wave packet (coherent superposition) or a delocalized vibrational eigenstate on the excited state PES. Different pumpdump schemes and the choice of the electronic excited state will be discussed elsewhere. ${ }^{72}$ For the present discussion we assume that the $\nu=4$ level of the $2 A^{\prime}$ PES, which lies $0.25 \mathrm{eV}$ above the excited state TS barrier and has significant Franck-Condon overlaps with vibrational eigenstates on both basins of the electronic ground state, is populated with a cw laser. This choice is not critical; the general features and trends hold for different choices, provided the initial wave packet or eigenstate in the excited electronic state is fairly delocalized.

\section{Monitoring real-time proton-transfer dynamics through photoelectron signals}

The $\nu=4$ level of the $2 A^{\prime}$ state was dumped with a Gaussian laser pulse with full width at half maximum (FWHM) of 40 fs and field intensity of 0.001 a.u. The upper panel of Fig. 7 shows the resulting population $\left(P_{d}\right)$ and wave-packet energy $\left(E_{d}\right)$ in the ground state for a range of dump frequencies. Populations above 0.1 can be achieved for $\hbar \omega_{d}>5.85 \mathrm{eV}$, though the Franck-Condon overlaps give rise to oscillations, especially for higher frequencies due to the increasing spacing between vibrational levels (higher dump frequencies imply lower vibrational energies). The leftpopulation ratio $\left(P_{\text {left }} / P_{d}\right)$, a measure of how much of the total dumped population is located to the left of the PT barrier, is shown as a function of time in the lower panel of
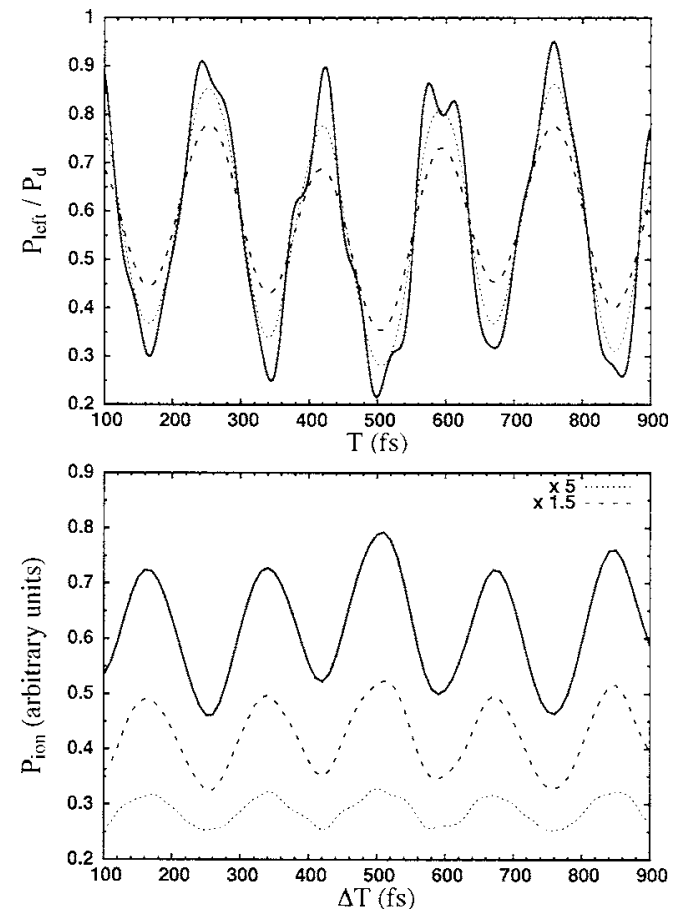

FIG. 8. Upper panel: left-population ratio for the dumped wave packet. Thick solid line: unconvoluted; dotted line: convoluted with FWHM $=40$ fs; dashed line: convoluted with FWHM=70 fs. Lower panel: total photoelectron signals $\left(P_{\text {ion }}\right)$ in arbitrary units for different probe pulse widths. For the sake of presentation, the signals for FWHM=10 and $40 \mathrm{fs}$ have been multiplied by 5 and 1.5, respectively. Dotted line: FWHM $=10$ fs; dashed line: $F W H M=40$ fs; solid line: $F W H M=70$ fs. In all cases the frequency of the probe pulse was $\hbar \omega_{p}=9.00 \mathrm{eV}$.

Fig. 7 for several frequencies. For $\hbar \omega_{d}=5.75$ and $5.95 \mathrm{eV}$ (dashed and thick solid lines, respectively) proton transfer takes place, though with different frequencies due to differences in wave-packet composition. For $\hbar \omega_{d}=6.10 \mathrm{eV}$ the energy of the dumped wave packet lies below the ground state barrier (see the upper panel of Fig. 7) and the probability remains constrained to the left $\left(P_{\text {left }} / P_{d}>0.5\right)$. Resonance conditions with localized vibrational eigenstates may also give rise to localized wave packets, but such details will be discussed elsewhere. ${ }^{72}$

Figure 8 shows the total photoelectron signals $\left(P_{\text {ion }}\right)$ for these dump-probe simulations with $\hbar \omega_{d}=5.95 \mathrm{eV}$ (lower panel) along with the time evolution of the left-population ratio in the ground state (upper panel). The period of PT is seen to be about $170 \mathrm{fs}$. To assess the effect of finite pulse widths on the left-population ratio, we also show these ratios convoluted over several Gaussian profiles with FWHM $=40$ and $70 \mathrm{fs}$ (the result of a $10 \mathrm{fs}$ convolution was hardly distinguishable from the unconvoluted population). Though some detail is lost at the maxima and minima, PT is still very evident in the convoluted populations. The lower panel of Fig. 8 shows photoelectron signals $\left(P_{\text {ion }}\right)$ for a probe frequency of $\hbar \omega_{p}=9.0 \mathrm{eV}$, which allows for ionization everywhere on the ground state PES, and for pulses with FWHM $=10,40$, and 70 fs. The ionization signals in Fig. 8 are shown in arbitrary units. The 10 fs pulse displays a richer structure which could be understood by arbitrarily switching off the transition moments in different regions, thus singling out photoelectron signals from only the transition state re- 
gion, as well as only the right and only the left basins. Ionization from the right basin is always more effective than from the left basin, giving rise to minima in the photoelectron signals close to the peaks of the left-population ratio. The structure between neighboring minima and maxima seen in the $10 \mathrm{fs}$ signal arises from the vicinity of the transition state. The weaker signals coming from the left basin could be understood on the basis of Franck-Condon factors, which favor ionization from the right basin. Detailed resolution of the photoelectron signals may be difficult as it would require very short probe pulses (FWHM $<10$ fs). Nevertheless, the $F W H M=40$ and 70 fs signals are clearly out of phase with the left-population ratio, indicating that intramolecular proton transfer could indeed be probed. Exploratory $a b$ initio studies underway indicate that use of $a b$ initio photoionization amplitudes enhances the difference between signals coming from the left and right basins. This should lead to richer ionization signals and permit the use of broader probe pulses.

\section{Ionization with geometry relaxation}

Though photoionization is generally well described in a sudden approximation, it is instructive, in the present context, to examine this process in the adiabatic limit. In this case, vibrational relaxation is viewed as rapid, leading to different skeleton geometries in the ion and ground states. To gain some insight into the effect of skeleton relaxation, we employed the procedure discussed in Sec. III B to generate a classically averaged potential surface for the ion state by integrating the trajectories on unrestricted HF (UHF) potentials. Though not shown here, the resulting ion potential surface is fairly similar to the previous one (upper panel of Fig. 7) with the main difference being a vertical shift of about $0.4 \mathrm{eV}$. One might hence expect the following dynamical picture. After vertical ionization (to the ground state average geometry) skeleton relaxation would bring the system into the ion average geometry, thus transferring about $0.4 \mathrm{eV}$ to proton vibrational energy. (Actually only some fraction of $0.4 \mathrm{eV}$ is expected to be transferred on the time scale of the probe pulse.) In view of the similar shapes of the ion potential surfaces, Franck-Condon factors still favor ionization from the right basin, and ion signals obtained with vertical and adiabatic approximations for the ion state geometry indeed show no qualitative difference.

\section{CONCLUDING REMARKS}

We have proposed and explored a simple and broadly applicable method for generating effective time-independent potential energy surfaces of reduced dimensionality, wherein the coupling between reaction and substrate modes is embodied by averaging over an ensemble of classical trajectories. These effective potentials should provide a more realistic description of the coupling between reaction and substrate modes than the usual sudden and adiabatic approximations for the molecular skeleton, commonly employed in studies of PT, and should facilitate simulations of the time-resolved photoelectron spectra of intramolecular PT in multidimensional systems. In applications the underlying effort should be comparable to that of the CRS/TDH sheme, which has proven useful where low-energy (ground state) initial conditions can be assumed. However, in intramolecular PT for energies around the transition state barrier, the CRS/TDH approach could run into difficulties due to the harmonic assumption for the bath modes. Some exploratory studies of classically averaged and CRS/TDH geometries suggest that the former can satisfactorily address higher vibrational energies in PT reactions. Use of a classically averaged substrate geometry also obviates difficulties that would arise when time-dependent effective potential schemes are employed in applications to time-resolved photoelectron spectroscopy. The reaction (proton) coordinates would then experience a time-dependent potential, and resonance conditions would be blurred.

We have explored the use of these classically averaged effective potentials in studies of time-resolved photoelectron spectra of intramolecular PT in chloromalonaldehyde, albeit assuming a constant dipole matrix element for photoionization of the wave packet on the ground state potential. These studies show that photoelectron signals clearly track wavepacket motion in the electronic ground state with varying level of detail for different widths of the probe pulse. Pumpdump-probe photoelectron spectra for proton transfer in this system, employing $a b$ initio values of the photoionization amplitudes, are underway and will be reported in a future publication. $^{72}$

\section{ACKNOWLEDGMENTS}

This work has been supported by Fundação de Amparo à Pesquisa do Estado de São Paulo-FAPESP (Brazil), by the National Science Foundation (U.S.), and by a Grant-in-Aid for Basic Science and the 21st Century COE Program for Frontier in Fundamental Chemistry from the Ministry of Education Science Sports and Culture of Japan.

${ }^{1}$ D. M. Neumark, Annu. Rev. Phys. Chem. 52, 255 (2001).

${ }^{2}$ See several review articles in Adv. Ser. Phys. Chem. 10A, (1999).

${ }^{3}$ T. Baumert, R. Thalweiser, V. Weiss, and G. Gerber, in Femtosecond Chemistry, edited by J. Manz and L. Wöste (VCH, Weinheim, 1995), Chap. 12.

${ }^{4}$ V. Blanchet, M. Z. Zgierski, T. Seideman, and A. Stolow, Nature (London) 401, 52 (1999).

${ }^{5}$ S. Lochbrunner, T. Schultz, M. Schmitt, J. P. Shaffer, M. Z. Zgierski, and A. Stolow, J. Chem. Phys. 114, 2519 (2001).

${ }^{6}$ M. Seel and W. Domcke, J. Chem. Phys. 95, 7806 (1991).

${ }^{7}$ Ch. Meier and V. Engel, J. Chem. Phys. 101, 2673 (1994).

${ }^{8}$ T. Suzuki and B. J. Whitaker, Int. Rev. Phys. Chem. 20, 313 (2001).

${ }^{9}$ M. Tsubouchi, B. J. Whitaker, L. Wang, H. Kohguchi, and T. Suzuki, Phys. Rev. Lett. 86, 4500 (2001).

${ }^{10}$ J. A. Davies, R. E. Continetti, D. W. Chandler, and C. C. Hayden, Phys. Rev. Lett. 84, 5983 (2000)

${ }^{11}$ Y. Arasaki, K. Takatsuka, K. Wang, and V. McKoy, Chem. Phys. Lett. 302, 363 (1999); J. Chem. Phys. 112, 8871 (2000).

${ }^{12}$ T. Seideman, J. Chem. Phys. 107, 7859 (1997).

${ }^{13}$ T. Seideman, Phys. Rev. A 64, 042504 (2001).

${ }^{14}$ Y. Arasaki, K. Takatsuka, K. Wang, and V. McKoy, Phys. Rev. Lett. 90, 248303 (2003); J. Chem. Phys. 119, 7913 (2003).

${ }^{15}$ T. Seideman, Annu. Rev. Phys. Chem. 53, 41 (2002).

${ }^{16}$ Y. Suzuki, M. Stener, and T. Seideman, J. Chem. Phys. 118, 4432 (2003).

${ }^{17}$ Y. Arasaki, K. Takatsuka, K. Wang, and V. McKoy, J. Chem. Phys. 114, 7941 (2001)

${ }^{18}$ S. C. Althorpe and T. Seideman, J. Chem. Phys. 110, 147 (1999).

${ }^{19}$ S. C. Althorpe and T. Seideman, J. Chem. Phys. 113, 7901 (2000). 
${ }^{20}$ A. Kühl and W. Domcke, Chem. Phys. 259, 227 (2000); J. Chem. Phys. 116, 263 (2002).

${ }^{21}$ Proton Transfer in Hydrogen-Bonded Systems, edited by T. Bountis (Plenum, New York, 1992).

${ }^{22}$ W. Klöpffer, Adv. Photochem. 10, 311 (1977); T. Werner, J. Phys. Chem. 83, 320 (1979).

${ }^{23}$ A. Douhal, F. Amat-Guerr, M. P. Lillo, and A. U. Acuña, J. Photochem. Photobiol., A 78, 127 (1994), and references therein.

${ }^{24}$ I. García-Ochoa, M. A. Díez López, M. H. Viñas, L. Santos, E. Martínez Ataz, F. Amat-Guerri, and A. Douhal, Chem.-Eur. J. 5, 897 (1999), and references therein.

${ }^{25}$ H. Ushiyama and K. Takatsuka, J. Chem. Phys. 115, 5903 (2001).

${ }^{26} \mathrm{H}$. Ushiyama and K. Takatsuka, Angew. Chem., Int. Ed. 44, 1237 (2005).

${ }^{27}$ J. Bicerano, H. F. SchaeferIII, and W. H. Miller, J. Am. Chem. Soc. 105, 2550 (1983).

${ }^{28}$ T. Carrington, Jr. and W. H. Miller, J. Chem. Phys. 84, 4364 (1986).

${ }^{29}$ N. Shida, P. F. Barbara, and J. E. Almlöf, J. Chem. Phys. 91, 4061 (1989).

${ }^{30}$ E. Bosch, M. Moreno, J. M. Lluch, and J. Bertrán, J. Chem. Phys. 93, 5685 (1990)

${ }^{31}$ T. D. Sewell, Y. Guo, and D. L. Thompson, J. Chem. Phys. 103, 8557 (1995).

${ }^{32}$ K. Yagi, T. Taketsugu, and K. Hirao, J. Chem. Phys. 115, 10647 (2001); G. V. Mil'nikov, K. Yagi, T. Taketsugu, H. Nakamura, and K. Hirao, ibid. 119, 10 (2003).

${ }^{33}$ V. A. Benderskii, E. V. Vetoshkin, I. S. Irgibaeva, and H. P. Tommsdorff, Chem. Phys. 262, 393 (2000); Russ. Chem. Bull. 50, 1148 (2002).

${ }^{34}$ D. Babić, S. D. Bosanac, and N. Došlić, Chem. Phys. Lett. 358, 337 (2002).

${ }^{35}$ C. S. Tautermann, A. F. Voegele, T. Loerting, and K. R. Liedl, J. Chem. Phys. 117, 1962 (2002); 117, 1967 (2002).

${ }^{36}$ S. F. Tayyari, M. Z. Tabrizi, F. Tayyari, and F. Milani-Nejad, J. Mol. Struct.: THEOCHEM 637, 171 (2003).

${ }^{37}$ M. Coutinho-Neto, A. Viel, and U. Manthe, J. Chem. Phys. 121, 9207 (2004).

${ }^{38}$ Z. Latajka and S. Scheiner, J. Phys. Chem. 96, 9764 (1992).

${ }^{39}$ K. Luth and S. Scheiner, J. Phys. Chem. 98, 3582 (1994).

${ }^{40}$ V. Barone and C. Adamo, J. Chem. Phys. 105, 11007 (1996).

${ }^{41}$ A. A. Arias, T. A. W. Wasserman, and P. H. Vaccaro, J. Chem. Phys. 107, 5617 (1997)

${ }^{42}$ A. L. Sobolewski and W. Domcke, J. Phys. Chem. A 103, 4494 (1999).

${ }^{43}$ T. Kar, S. Scheiner, and M. Čuma, J. Chem. Phys. 111, 849 (1999).

${ }^{44}$ S. Scheiner, J. Phys. Chem. A 104, 5898 (2000).

${ }^{45}$ A. H. Zewail, J. Phys. Chem. A 104, 5660 (2000).

${ }^{46}$ K. Fuke, H. Yoshiuchi, and K. Kaya, J. Phys. Chem. 88, 5840 (1984); K. Fuke and K. Kaya, ibid. 93, 614 (1989); A. Douhal, S. K. Kim, and A. H. Zewail, Nature (London) 378, 260 (1995); T. Fiebig, M. Chachisvilis, M. M. Manger, and A. H. Zewail, J. Phys. Chem. A 103, 7419 (1999); S Takeuchi and T. Tahara, J. Phys. Chem. 102, 7740 (1998); Chem. Phys. Lett. 277, 340 (1997); Chem. Phys. Lett. 347, 108 (2001).
${ }^{47}$ H. Ishikawa, K. Iwata, and H. Hamaguchi, J. Phys. Chem. A 106, 2305 (2002).

${ }^{48}$ Y. Arasaki, K. Yamazaki, M. T. do N. Varella, and K. Takatsuka, Chem. Phys. 311, 255 (2005).

${ }^{49}$ B. A. Ruf and W. H. Miller, J. Chem. Soc., Faraday Trans. 2 84, 1523 (1988).

${ }^{50}$ G. K. Paramonov, H. Naundorf, and O. Kühn, Eur. Phys. J. D 14, 205 (2001).

${ }^{51}$ H. Naundorf, G. A. Worth, H.-D. Meyer, and O. Kühn, J. Phys. Chem. A 106, 719 (2002).

${ }^{52}$ H. Naundorf, J. A. Organero, A. Douhal, and O. Kühn, J. Chem. Phys. 110, 11286 (1999).

${ }^{53}$ R. Xu, Y. J. Yan, and O. Kühn, Eur. Phys. J. D 19, 293 (2002).

${ }^{54}$ Use of a dump-probe scheme should suffice here where the focus is on surfaces to be employed in applications to time-resolved photoelectron spectroscopy. Extension to a pump-dump-probe scheme, where the proton wave packet is initially in the electronic ground state, is straightforward and will be the subject of a future publication (Ref. 72).

${ }^{55}$ D. Kosloff and R. Kosloff, J. Comput. Phys. 52, 35 (1983); R. Kosloff, J. Phys. Chem. 92, 2087 (1988).

${ }^{56}$ K. Takahashi and K. Ikeda, J. Chem. Phys. 99, 8680 (1993).

${ }^{57}$ M. H. Beck, A. Jäckle, G. A. Worth, and H.-D. Meyer, Phys. Rep. 324, 1 (2000)

${ }^{58}$ M. Petković and O. Kühn, J. Phys. Chem. A 107, 8458 (2003).

${ }^{59}$ K. Giese, D. Lahav, and O. Kühn, J. Theor. Comput. Chem. 3, 567 (2004).

${ }^{60}$ K. Giese, H. Ushiyama, K. Takatsuka, and O. Kühn, J. Chem. Phys. 122, 124307 (2005).

${ }^{61}$ K. Husimi, Prog. Theor. Phys. 9, 381 (1953).

${ }^{62}$ K. Wolf, W. Mikenda, E. Nusterer, K. Schwarz, and C. Ullbricht, Chem.Eur. J. 4, 1418 (1998).

${ }^{63}$ K. Wolf, W. Mikenda, E. Nusterer, and K. Schwarz, J. Mol. Struct. 448, 201 (1998).

${ }^{64}$ O. A. Sharafeddin, K. Hinsen, T. Carrington, Jr., and B. Roux, J. Comput. Chem. 18, 1760 (1997).

${ }^{65}$ P. Jungwirth and R. B. Gerber, J. Chem. Phys. 102, 6046 (1995).

${ }^{66}$ P. Jungwirth and R. B. Gerber, J. Chem. Phys. 102, 8855 (1995).

${ }^{67}$ P. Jungwirth, E. Fredj, and R. B. Gerber, J. Chem. Phys. 104, 9332 (1996).

${ }^{68}$ R. B. Gerber, P. Jungwirth, E. Fredj, and A. Y. Rom, in Modern Methods for Multidimensional Dynamics Computations in Chemistry, edited by D. L. Thompson (World Scientific, Singapore, 1998), p. 238.

${ }^{69}$ D. Lauvergnat and A. Nauts, J. Chem. Phys. 116, 8560 (2002).

${ }^{70}$ M. W. Schmidt, K. K. Baldridge, J. A. Boatz et al., J. Comput. Chem. 14, 1347 (1993).

${ }^{71}$ H. Ushiyama, Y. Arasaki, and K. Takatsuka, Chem. Phys. Lett. 346, 169 (2001).

${ }^{72}$ M. T. do N. Varella, H. Ushiyama, Y. Arasaki, K. Takatsuka, K. Wang, and V. McKoy (unpublished) 